\title{
On the Compactness of the Commutator of the Parabolic Marcinkiewicz Integral with Variable Kernel
}

\author{
Yanping Chen, ${ }^{1}$ Kai Zhu, ${ }^{1}$ and Yong Ding ${ }^{2}$ \\ ${ }^{1}$ Department of Applied Mathematics, School of Mathematics and Physics, University of Science and Technology Beijing, \\ Beijing 100083, China \\ ${ }^{2}$ Laboratory of Mathematics and Complex Systems (BNU), School of Mathematical Sciences, Beijing Normal University, \\ Ministry of Education, Beijing 100875, China
}

Correspondence should be addressed to Kai Zhu; kaizhu0116@126.com

Received 30 June 2014; Accepted 17 October 2014; Published 12 November 2014

Academic Editor: Gestur Ólafsson

Copyright (C) 2014 Yanping Chen et al. This is an open access article distributed under the Creative Commons Attribution License, which permits unrestricted use, distribution, and reproduction in any medium, provided the original work is properly cited.

The authors prove that the commutator of the parabolic Marcinkiewicz integral $g_{\Omega}$ with variable kernel is a compact operator on $L^{p}\left(\mathbb{R}^{n}\right)(1<p<\infty)$ if and only if $b \in \mathrm{CMO}\left(\mathbb{R}^{n}, \rho\right)$. The result is substantial improvement and extension of some known results.

\section{Introduction and Main Results}

Let $\mathbb{R}^{n}$ be the $n$-dimension Euclidean space. Let $S^{n-1}=\{x \in$ $\left.\mathbb{R}^{n}:|x|=1\right\}$ be the unit sphere on $\mathbb{R}^{n}$ equipped with the Lebesgue measure $d \sigma\left(x^{\prime}\right)$. For $b \in L_{\mathrm{loc}}\left(\mathbb{R}^{n}\right)$, denote by $m_{b}$ the multiplication operator defined by $m_{b} f=b f$ for the measurable function $f$. Suppose that $A$ is a linear operator on some measurable function space; then the commutator formed by $A$ and $m_{b}$ is defined by

$$
\begin{aligned}
{[b, A] f(x) } & :=m_{b}(A f)(x)-A\left(m_{b} f\right)(x) \\
& =b(x) A f(x)-A(b f)(x) .
\end{aligned}
$$

A famous theorem of Coifman et al. [1] characterized the $L^{p_{-}}$ boundedness of $\left[b, R_{j}\right]$, where $R_{j}(j=1, \ldots, n)$ are the Riesz transforms and $b \in \mathrm{BMO}\left(\mathbb{R}^{n}\right)$. Using this characterization, the authors of [1] got a decomposition theorem of the real Hardy space $H^{1}\left(\mathbb{R}^{n}\right)$. Uchiyama [2] and Janson [3] showed that the Riesz transform $R_{j}$ may be replaced by the CalderónZygmund singular integral operator $T_{\Omega}$. Moreover, in 1978, Uchiyama [2] gave a characterization of the compactness for the commutator $\left[b, T_{\Omega}\right]$ defined by

$$
\left[b, T_{\Omega}\right] f(x)=\text { p.v. } \int_{\mathbb{R}^{n}}[b(x)-b(y)] \frac{\Omega(x-y)}{|x-y|^{n}} f(y) d y,
$$

where $\Omega$ is homogeneous function of degree zero on $\mathbb{R}^{n} \backslash\{0\}$, that is,

$$
\Omega(t x)=\Omega(x) \text { for any } t>0, x \in \mathbb{R}^{n} \backslash\{0\},
$$

and $\Omega$ has mean zero on $S^{n-1}$, the unit sphere in $\mathbb{R}^{n}$, that is,

$$
\int_{S^{n-1}} \Omega\left(x^{\prime}\right) d \sigma\left(x^{\prime}\right)=0 .
$$

Theorem A (see [2]). Suppose that $\Omega \in \operatorname{Lip}\left(S^{n-1}\right)$ satisfies (3) and (4). Let $b \in L_{\mathrm{loc}}\left(\mathbb{R}^{n}\right)$.

(i) If $\left[b, T_{\Omega}\right]$ is a compact operator on $L^{p}\left(\mathbb{R}^{n}\right)$ for some $p$, $1<p<\infty$, then $b \in C M O\left(\mathbb{R}^{n}\right)$.

(ii) If $b \in C M O\left(\mathbb{R}^{n}\right)$, then $\left[b, T_{\Omega}\right]$ is a compact operator on $L^{p}\left(\mathbb{R}^{n}\right)$ for $1<p<\infty$, where $C M O\left(\mathbb{R}^{n}\right)$ is the $B M O$-closure of $C_{c}^{1}\left(\mathbb{R}^{n}\right)$ and $C_{c}^{1}\left(\mathbb{R}^{n}\right)$ denotes the set of $C^{1}$-functions with compact support in $\mathbb{R}^{n}$.

The interest in the compactness of $\left[b, T_{\Omega}\right]$ in complex analysis is from the connection between the commutators and the Hankel-type operators. In fact, Beatrous and Li [4] proved the boundedness and compactness of $\left[b, T_{\Omega}\right]$ on $L^{p}$ over some spaces of homogeneous type. Krantz and Li (see [5]) have applied the characterization of $L^{p}$-compactness of commutator to give a compactness of Hankel operators on 
holomorphic Hardy spaces $H^{2}(D)$, where $D$ is a bounded, strictly pseudoconvex domain in $\mathbb{C}^{n}$.

In this paper, we will study the compactness of the commutator of the parabolic Marcinkiewicz integral with variable kernel. Let us recall the definition of parabolic metric. Let $\alpha_{1}, \ldots, \alpha_{n}$ be fixed real numbers, $\alpha_{i} \geq 1$. For fixed $x=\left(x_{1}, \ldots, x_{n}\right) \in \mathbb{R}^{n}$, the function $F(x, \rho)=\sum_{i=1}^{n}\left(x_{i}^{2} / \rho^{2 \alpha_{i}}\right)$ is a decreasing function in $\rho(\rho>0)$. We denote by $\rho(x)$ the unique solution of the equation $F(x, \rho)=1$. In 1966, Fabes and Rivière [6] proved that $\rho(x)$ is a metric on $\mathbb{R}^{n}$. For $\mu>0$ and $x \in \mathbb{R}^{n}$, define the dilation on $\mathbb{R}^{n}$ by

$$
A_{\mu}:\left(x_{1}, x_{2}, \ldots, x_{n}\right) \longrightarrow\left(\mu^{\alpha_{1}} x_{1}, \mu^{\alpha_{2}} x_{2}, \ldots, \mu^{\alpha_{n}} x_{n}\right)
$$

Then it is immediate to see that $\rho\left(A_{\mu} x\right)=\mu \rho(x)$ and $\rho(t x) \leq$ $\rho(x)$ when $t \leq 1$. One has the polar decomposition $x=A_{\rho} x^{\prime}$ with $x^{\prime}=\left(x_{1} / \rho(x)^{\alpha_{1}}, \ldots, x_{n} / \rho(x)^{\alpha_{n}}\right) \in S^{n-1}, \rho=\rho(x)$ and $d x=\rho^{\alpha-1} J\left(x^{\prime}\right) d \rho d \sigma\left(x^{\prime}\right)$, where $\alpha=\sum_{i=1}^{n} \alpha_{i}, J\left(x^{\prime}\right)$ is a $C^{\infty}$ function on $S^{n-1}$ and bounded below uniformly by 1 .

In 1974, Madych [7] gave the $L^{p}(1<p<\infty)$ boundedness of the parabolic Marcinkiewicz integral $g_{\psi}$ with respect to the transform $A_{t}$, where

$$
g_{\psi}(f)(x)=\left(\int_{0}^{\infty}\left|\psi_{t} * f(x)\right|^{2} \frac{d t}{t}\right)^{1 / 2}
$$

with $\psi \in S\left(\mathbb{R}^{n}\right)$ satisfying $\int_{\mathbb{R}^{n}} \psi(x) d x=0$, and $\psi_{t}(x)=$ $t^{-\alpha} \psi\left(A_{t-1} x\right)$ for $t>0$. Inspired by the works in [6,7], recently, Xue et al. [8] improved the above result. More precisely, the authors in [8] proved that the parabolic Marcinkiewicz integral is still bounded on $L^{p}$ if replacing $\psi(x)$ by a kernel function $\phi(x)=\Omega(x) \rho(x)^{-\alpha+1} \chi_{\{\rho(x) \leq 1\}}(x)$ with $\Omega \in$ $L^{q}\left(S^{n-1}\right)(q>1)$ satisfying the following conditions:

$$
\begin{gathered}
\Omega\left(A_{\mu} z\right)=\Omega(z), \quad \text { for any } z \in \mathbb{R}^{n} \backslash\{0\}, \mu>0, \\
\int_{S^{n-1}} \Omega\left(z^{\prime}\right) J\left(z^{\prime}\right) d \sigma\left(z^{\prime}\right)=0 .
\end{gathered}
$$

Afterwards, many authors studied the properties of the parabolic Marcinkiewicz integral and its commutators which is defined by

$$
g_{\psi ; b}(f)(x)=\left(\int_{0}^{\infty}\left|\left[b, \psi_{t}\right] f(x)\right|^{2} \frac{d t}{t}\right)^{1 / 2}
$$

(see, e.g., [9-12]).

Notice that if $\alpha_{1}=\cdots=\alpha_{n}=1$, then $\rho(x)=|x|$ and $\left(\mathbb{R}^{n}, \rho\right)=\left(\mathbb{R}^{n},|\cdot|\right)$. In this case, $g_{\psi}$ and $g_{\psi ; b}$ are just the classical Marcinkiewicz integral and it is commutators which play a very important role in the singular integral operators theory, function spaces theory, and PDE (see, e.g., [13-23]).

Let us turn to the definitions of the parabolic Marcinkiewicz integral with variable kernel and its commutator. Suppose that $\Omega(x, z)$ satisfies the following conditions:

$$
\begin{gathered}
\Omega\left(x, A_{\mu} z\right)=\Omega(x, z), \\
\text { for any } x \in \mathbb{R}^{n}, \quad z \in \mathbb{R}^{n} \backslash\{0\}, \quad \mu>0, \\
\int_{S^{n-1}} \Omega\left(x, z^{\prime}\right) J\left(z^{\prime}\right) d \sigma\left(z^{\prime}\right)=0, \quad \text { for any } x \in \mathbb{R}^{n} .
\end{gathered}
$$

Then the parabolic Marcinkiewicz integral with variable kernel is defined by

$$
g_{\Omega}(f)(x)=\left\{\int_{0}^{\infty}\left|\int_{\rho(x-y) \leq t} \frac{\Omega(x, x-y)}{\rho(x-y)^{\alpha-1}} f(y) d y\right|^{2} \frac{d t}{t^{3}}\right\}^{1 / 2} .
$$

Let us now give the definition of parabolic BMO space. Denote by $\mathscr{E}(x, r)$ the ellipsoid center at $x$ and radius $r$. More precisely, $\mathscr{E}(x, r)=\{y: \rho(x-y)<r\}$. For $j>0$, we denote $j \mathscr{E}(x, r)$ to be the $j$-times extension of $\mathscr{E}(x, r)$ with the same center. That is, $j \mathscr{E}(x, r)=\{y: \rho(x-y)<j r\}$. Moreover, $|\mathscr{E}(x, r)|$ stands the Lebesgue measure of $\mathscr{E}(x, r)$, which is comparable to $r^{\alpha}$. $\mathscr{E}^{c}$ denotes the complement of $\mathscr{E}$.

For a measurable and locally integrable function $b$ : $\mathbb{R}^{n} \rightarrow \mathbb{R}$ set

$$
\eta_{b}(\theta)=\sup _{r \leq \theta} \frac{1}{|\mathscr{E}(r)|} \int_{\mathscr{E}(r)}\left|b(y)-b_{\mathscr{E}(r)}\right| d y \quad \text { for } \theta>0,
$$

where $\mathscr{E}(r)$ is any ellipsoid in $\mathbb{R}^{n}$ of radius $r$ and $b_{\mathscr{E}(r)}=$ $|\mathscr{E}(r)|^{-1} \int_{\mathscr{E}(r)} b(y) d y$ is the average of $b$ on $\mathscr{E}(r)$. We say $b \in$ $\operatorname{BMO}\left(\mathbb{R}^{n}, \rho\right)$ if $\|b\|_{*, \rho}:=\sup _{\theta} \eta_{b}(\theta)<\infty$ (see [24]).

Let $b \in L_{\text {loc }}\left(\mathbb{R}^{n}\right)$; the commutator of the parabolic Marcinkiewicz integral with variable kernel is defined by

$g_{\Omega ; b} f(x)$

$$
=\left\{\int_{0}^{\infty}\left|\int_{\rho(x-y) \leq t} \frac{\Omega(x, x-y)}{\rho(x-y)^{\alpha-1}}(b(x)-b(y)) f(y) d y\right|^{2} \frac{d t}{t^{3}}\right\}^{1 / 2} .
$$

Notice that if $\alpha_{1}=\cdots=\alpha_{n}=1$, then $\rho(x)=|x| ; g_{\Omega}$ and $g_{\Omega ; b}$ are the classical Marcinkiewicz integral with variable kernel and the classical commutator of Marcinkiewicz integral with variable kernel which was studied by many authors (see, e.g., [25-28]).

In 2009, Chen and Ding [29] studied the parabolic Marcinkiewicz integral with variable kernel and its commutator on the Lebesgue space $L^{p}\left(\mathbb{R}^{n}\right)$, which is stated as follows.

Theorem B. Let $1<p<\infty$. Suppose that $\Omega(x, z)$ satisfies (9), (10) and for every multi-index $\beta$,

$$
\sup _{\substack{x \in \mathbb{R}^{n} \\ \xi^{\prime} \in S^{n-1}}}\left|D_{\xi}^{\beta} \Omega\left(x, \xi^{\prime}\right)\right| \leq C(\beta) .
$$

Let $b \in B M O\left(\mathbb{R}^{n}, \rho\right)$. Then there exists a constant $C>0$ such that, for any $f \in L^{p}\left(\mathbb{R}^{n}\right)$,

$$
\left\|g_{\Omega ; b} f\right\|_{p} \leq C\|b\|_{*, \rho}\|f\|_{p} .
$$

In this paper, we will give a characterization of the commutator of the parabolic Marcinkiewicz integral with variable kernel which is compact operator on $L^{p}\left(\mathbb{R}^{n}\right)(1<$ $p<\infty)$. First, we will prove that the commutator of the parabolic Marcinkiewicz integral with variable kernel is bounded on $L^{p}\left(\mathbb{R}^{n}\right)(1<p<\infty)$. To show our result, we give the following definition. 
Definition 1 (see [30]). Let $X$ and $Y$ be Banach spaces and let $U$ be a subset of $X$. Then $T: U \mapsto Y$ is compact if $T$ is continuous and maps bounded subsets of $U$ into strongly precompact subsets of $Y$.

Our first result can be stated as follows.

Theorem 2. Let $\Omega(x, z)$ satisfy (9), (10), (14) and for any multiindex $\beta$

$$
\sup _{\xi^{\prime} \in S^{n-1}}\left|D_{\xi}^{\beta} \Omega\left(x, \xi^{\prime}\right)-D_{\xi}^{\beta} \Omega\left(y, \xi^{\prime}\right)\right| \leq C \rho(x-y) .
$$

If $b \in C M O\left(\mathbb{R}^{n}, \rho\right)$, where $C M O\left(\mathbb{R}^{n}, \rho\right)$ is the $B M O\left(\mathbb{R}^{n}, \rho\right)$ closure of $C_{c}^{1}\left(\mathbb{R}^{n}\right)$ and $C_{c}^{1}\left(\mathbb{R}^{n}\right)$ denotes the set of $C^{1}$-functions with compact support in $\mathbb{R}^{n}$, then $g_{\Omega ; b}$ is a compact operator on $L^{p}\left(\mathbb{R}^{n}\right)(1<p<\infty)$.

On the other hand, we will give the converse part of the compactness of the commutator of the Marcinkiewicz integral with variable kernel.

Theorem 3. Suppose that $\Omega$ satisfies (9) and (10),

$$
\begin{array}{r}
\sup _{z \in \mathbb{R}^{n}}\left|\Omega\left(z, x^{\prime}\right)-\Omega\left(z, y^{\prime}\right)\right| \leq \rho\left(x^{\prime}-y^{\prime}\right), \\
\text { for } x^{\prime}, y^{\prime} \in S^{n-1},
\end{array}
$$

and there exists $\Theta \subset S^{n-1}, 0<\delta<1$ such that, for any $x \in \mathbb{R}^{n}$,

$$
\sigma(\Theta)=\sigma\left(\left\{y^{\prime} \in S^{n-1}: \inf _{x \in \mathbb{R}^{n}} \Omega\left(x, y^{\prime}\right) \geq 2 \delta\right\}\right)>0,
$$

where $\sigma$ is the measure on $S^{n-1}$ which is induced from the Lebesgue measure on $\mathbb{R}^{n}$. If $\left[b, g_{\Omega}\right]$ is a bounded operator on $L^{p}\left(\mathbb{R}^{n}\right)$ for some $1<p<\infty$, then $b \in B M O\left(\mathbb{R}^{n}, \rho\right)$.

Theorem 4. Let $\Omega(x, z)$ satisfy the same conditions as in Theorem 2. If $g_{\Omega ; b}$ is a compact operator on $L^{p}\left(\mathbb{R}^{n}\right)$ for some $p(1<p<\infty)$, then $b \in C M O\left(\mathbb{R}^{n}, \rho\right)$.

Remark 5. Our results are still new even for the case $\rho(x)=$ $|x|$.

This paper is organized as follows. First, in Section 2, we give some important notations and lemmas, which will be used in the proofs of the main results. In Section 3, we prove Theorem 2. The proof of Theorem 3 is given in Section 4. In Section 5, we will give the proof of Theorem 4 .

The letter $C$ in the paper denotes positive constants independent of essential variables. For $p \geq 1, p^{\prime}$ denotes the dual exponent of $p$, that is, $p^{\prime}=p /(p-1)$.

\section{Notations and Lemmas}

For a given measurable function $f \in L_{\text {loc }}\left(\mathbb{R}^{n}\right)$, the HardyLittlewood maximal operator $\mathscr{M}$ and the sharp maximal operator $\mathscr{M}^{\#}$ related to ellipsoids are defined by

$$
\begin{gathered}
\mathscr{M} f(x)=\sup _{\mathscr{E} \ni x} \frac{1}{|\mathscr{E}|} \int_{\mathscr{E}}|f(y)| d y, \\
\mathscr{M}^{\#} f(x)=\sup _{\mathscr{E} \ni x} \frac{1}{|\mathscr{E}|} \int_{\mathscr{E}}\left|f(y)-f_{\mathscr{E}}\right| d y,
\end{gathered}
$$

respectively. Define also the operator $\mathscr{M}_{q} f(x)$ := $\left(\mathscr{M}\left(|f|^{q}\right)(x)\right)^{1 / q}$ for $1 \leq q<\infty$.

Denote by $H_{m}$ the space of all $n$-dimensional spherical harmonic of degree $m$ with its dimension $D_{m}=\operatorname{dim} H_{m}$. By $[24,31]$, we have

$$
D_{m} \leq C(n) m^{n-2}, \quad m \geq 1 .
$$

Furthermore, let $\left\{Y_{m, s}\left(x^{\prime}\right)\right\}_{s=1}^{D_{m}}$ be an orthonormal base of $H_{m}$. Then $\left\{Y_{m, s}\left(x^{\prime}\right)\right\}_{s=1}^{D_{m}}(m=0,1, \ldots)$ is a complete orthonormal system in $L^{2}\left(S^{n-1}\right)$ and for any multi-index $\beta$ (see $[24,31]$ ),

$$
\sup _{x^{\prime} \in S^{n-1}}\left|D_{x^{\prime}}^{\beta} Y_{m, s}\left(x^{\prime}\right)\right| \leq C(n) m^{|\beta|+(n-2) / 2}, \quad m=1,2, \ldots .
$$

If, for instance, $\phi \in C^{\infty}\left(S^{n-1}\right)$, then $\sum_{m} \sum_{s} a_{m, s} Y_{m, s}\left(x^{\prime}\right)$ is the Fourier series expansion of $\phi\left(x^{\prime}\right)$ with respect to $\left\{Y_{m, s}\left(x^{\prime}\right)\right\}_{m, s}$, where

$$
a_{m, s}=\int_{S^{n-1}} \phi\left(y^{\prime}\right) Y_{m, s}\left(y^{\prime}\right) d \sigma\left(y^{\prime}\right)
$$

and (see $[24,31])$

$$
\begin{aligned}
a_{m, s}= & (-1)^{l} m^{-l}(m+n-2)^{-l} \\
& \times \int_{S^{n-1}} L^{l}(\phi) Y_{m, s}\left(x^{\prime}\right) d \sigma\left(x^{\prime}\right), \quad m \geq 1,
\end{aligned}
$$

where $L(\phi)=|x|^{2} \Delta \phi(x), L^{l}(\phi)=L\left(L^{l-1}(\phi)\right)$ for any integer l. In particular, the expansion of $\phi$ into spherical harmonics converges uniformly to $\phi$. The next results will be employed in the forthcoming considerations.

Lemma 6 (see [29]). Suppose that $\mathscr{E}=\mathscr{E}\left(x_{0}, r\right)$. Let $\gamma>0$. Then

$$
\begin{aligned}
& \left|\frac{Y_{m, s}\left((x-y)^{\prime}\right)}{J\left((x-y)^{\prime}\right) \rho(x-y)^{\gamma}}-\frac{Y_{m, s}\left(\left(x_{0}-y\right)^{\prime}\right)}{J\left(\left(x_{0}-y\right)^{\prime}\right) \rho\left(x_{0}-y\right)^{\gamma}}\right| \\
& \quad \leq C m^{n / 2} \frac{\rho\left(x-x_{0}\right)}{\rho\left(y-x_{0}\right)^{\gamma+1}},
\end{aligned}
$$

for each $x \in \mathscr{E}$ and $y \in(2 \mathscr{E})^{c}$, where $x^{\prime}=\left(x_{1} / \rho(x)^{\alpha_{1}}, \ldots\right.$, $\left.x_{n} / \rho(x)^{\alpha_{n}}\right)$. 
Lemma 7 (Frechet-Kolmogorov, see [32, 33]). A subset $G$ of $L^{p}\left(\mathbb{R}^{n}\right), 1<p<\infty$, is strongly precompact if and only if it satisfies the conditions

$$
\begin{gathered}
\sup _{f \in G}\|f\|_{p}<\infty ; \\
\lim _{\rho(y) \rightarrow 0}\|f(\cdot+y)-f(\cdot)\|_{p}=0 \quad \text { uniformly in } f \in G ; \\
\lim _{\beta \rightarrow \infty}\left\|f \chi_{E_{\beta}}\right\|_{p}=0 \quad \text { uniformly in } f \in G, \\
\text { where } E_{\beta}=\left\{x \in \mathbb{R}^{n}: \rho(x)>\beta\right\} .
\end{gathered}
$$

Lemma 8 (see [29]). Suppose that $\mathscr{E}=\mathscr{E}\left(x_{0}, r\right)$. Let $\gamma>0$. Then

$$
\left|\frac{1}{\rho(x-y)^{\gamma}}-\frac{1}{\rho\left(x_{0}-y\right)^{\gamma}}\right| \leq 2\left(2^{\gamma}-1\right) \frac{\rho\left(x-x_{0}\right)}{\rho\left(y-x_{0}\right)^{\gamma+1}},
$$

for each $x \in \mathscr{E}$ and $y \in(2 \mathscr{E})^{c}$. (Here and below, for $a>0$ and $\mathscr{E}\left(x_{0}, r\right), a \mathscr{E}$ denotes the a-times extension of $\mathscr{E}$ with the same center, that is, $\left.a \mathscr{E}=\left\{y: \rho\left(y-x_{0}\right)<a r\right\}.\right)$

Lemma 9 (see [32]). If $\rho(x) \geq 4 \rho(y)$, then

$$
\rho\left((x-y)^{\prime}-x^{\prime}\right) \leq 3 \frac{\rho(y)}{\rho(x)},
$$

where $x^{\prime}=\left(x_{1} / \rho(x)^{\alpha_{1}}, \ldots, x_{n} / \rho(x)^{\alpha_{n}}\right) \in S^{n-1}$.

Lemma 10 (see $[2,32])$. If $b \in B M O\left(\mathbb{R}^{n}, \rho\right), C_{5}>C_{4}>2$, $\mathscr{E}$ is an ellipsoid centered at $x_{0}$ and radius $r$, then there exist positive constants $C_{6}, C_{7}$, and $C_{8}$ (depending on $C_{4}, C_{5}$, and b), such that

$$
\begin{aligned}
\mid\left\{C_{4} r\right. & \left.<\rho\left(x-x_{0}\right)<C_{5} r:\left|b(x)-b_{\mathscr{E}}\right|>v+C_{6}\right\} \mid \\
& \leq C_{7}|\mathscr{E}| e^{-C_{8} v}, \quad(0<v<\infty) .
\end{aligned}
$$

Lemma 11 (see [34]). Suppose that $f(x)$ is a measurable function, $\lambda_{f}(w)=\left|\left\{x \in \mathbb{R}^{n}:|f(x)|>w>0\right\}\right|$, and $E$ is a measurable set. Define $f^{*}(t)=\inf \left\{w: \lambda_{f}(w) \leq t\right\}$ for $t>0$; then

$$
\int_{E}|f(x)|^{p} d x \leq \int_{0}^{|E|}\left|f^{*}(t)\right|^{p} d t, \quad 1 \leq p<\infty .
$$

Lemma 12 (see $[2,32])$. Let $b \in B M O\left(\mathbb{R}^{n}, \rho\right)$. Then $b \in$ $C M O\left(\mathbb{R}^{n}, \rho\right)$ if and only if $b$ satisfies the following three conditions:

(i) $\lim _{a \rightarrow 0} \sup _{|\mathscr{E}|=a} M(b, \mathscr{E})=0$,

(ii) $\lim _{a \rightarrow \infty} \sup _{|\mathscr{E}|=a} M(b, \mathscr{E})=0$,

(iii) $\lim _{x \rightarrow \infty} M(b, \mathscr{E}+x)=0$, for each $\mathscr{E}$, where $M(b, \mathscr{E})=$ $(1 /|\mathscr{E}|) \int_{\mathscr{E}}\left|b(y)-b_{\mathscr{E}}\right| d y$.

It is easy to get the following lemma.
Lemma 13. If $\Omega$ satisfies conditions (9) and (17), then for $\beta>$ 0 ,

$$
\left|\frac{\Omega(z, x-y)}{\rho(x-y)^{\beta}}-\frac{\Omega(z, x)}{\rho(x)^{\beta}}\right| \leq C \frac{\rho(y)}{\rho(x)^{\beta+1}}
$$

for $\rho(x) \geq 4 \rho(y)$.

\section{Proof of Theorem 2}

Suppose that $b \in \operatorname{CMO}\left(\mathbb{R}^{n}, \rho\right)$. Then, by Theorem A, we can get

$$
\left\|g_{\Omega ; b} f\right\|_{p} \leq C\|b\|_{*, \rho}\|f\|_{p}, \quad \text { for any } f \in L^{p}\left(\mathbb{R}^{n}\right) .
$$

Then we can easily get $g_{\Omega ; b}$ is continuous on $L^{p}\left(\mathbb{R}^{n}\right)$, so by Definition 1, it suffices to prove that, for any bounded set $F$ in $L^{p}\left(\mathbb{R}^{n}\right), G=\left\{g_{\Omega ; b} f: f \in F\right\}$ is strongly precompact in $L^{p}\left(\mathbb{R}^{n}\right)$. Since for any $\varepsilon>0$ there exists $b^{\varepsilon} \in C_{0}^{\infty}$ such that $\left\|b-b^{\varepsilon}\right\|_{*, \rho}<\varepsilon$, then by (33),

$$
\left\|g_{\Omega ; b}-g_{\Omega ; b^{\varepsilon}}\right\|_{L^{p} \mapsto L^{p}} \leq\left\|g_{\Omega ; b-b^{\varepsilon}}\right\|_{L^{p} L^{p}}<C \varepsilon .
$$

Thus, it suffices to prove that $G$ is strongly precompact in $L^{p}\left(\mathbb{R}^{n}\right)$ for $b \in C_{c}^{\infty}\left(\mathbb{R}^{n}\right)$. By Lemma 7 , we need only to verify that (25)-(27) hold uniformly in $G$.

Suppose that $\|f\|_{p} \leq D$ for every $f \in F$. Notice that $b \in$ $C_{c}^{\infty}\left(\mathbb{R}^{n}\right)$ and applying (33), we have

$$
\sup _{f \in F}\left\|g_{\Omega ; b} f\right\|_{p} \leq C\|b\|_{*, \rho} \sup _{f \in F}\|f\|_{p} \leq C^{\prime} D<\infty .
$$

On the other hand, for any $\varepsilon>0$, we may take $B>0$ large enough such that

$$
\left(\int_{\rho(x)>B} \rho(x)^{-\alpha p} d x\right)^{1 / p}<\varepsilon .
$$

Assume $\operatorname{supp}(b) \subset\{y: \rho(y)<r\}$ for some $r>0$. Thus, for any $x$ satisfying $\rho(x)>\max \{B, 4 r\}$ and every $f \in F$, then by $\Omega \in L^{\infty}\left(\mathbb{R}^{n}\right) \times L^{\infty}\left(S^{n-1}\right)$ and $\rho(x-y) \sim \rho(x)$, we have

$$
\begin{aligned}
& \left|g_{\Omega ; b} f(x)\right| \\
& =\left\{\int_{0}^{\infty}\left|\int_{\rho(x-y) \leq t} \frac{\Omega(x, x-y)}{\rho(x-y)^{\alpha-1}}(b(x)-b(y)) f(y) d y\right|^{2} \frac{d t}{t^{3}}\right\}^{1 / 2} \\
& \leq C \int_{\operatorname{supp} b} \frac{|b(y)|}{\rho(x-y)^{\alpha-1}}|f(y)|\left\{\int_{\rho(x-y) \leq t} \frac{d t}{t^{3}}\right\}^{1 / 2} d y \\
& \leq C \rho(x)^{-\alpha}\|f\|_{p} .
\end{aligned}
$$

Applying (36), we have

$$
\left(\int_{\rho(x)>B}\left|g_{\Omega ; b} f(x)\right|^{p} d x\right)^{1 / p} \leq C \varepsilon .
$$


Equation (38) shows that (27) holds uniformly in G. Finally, to finish the proof of Theorem 2, it remains to show (26) holds uniformly in $G$. We need to prove that, for any $\varepsilon>0$, if $\rho(z)$ is sufficiently small, then for every $f \in F$,

$$
\left\|g_{\Omega ; b} f(\cdot)-g_{\Omega ; b} f(\cdot+z)\right\|_{p} \leq C \varepsilon .
$$

To do this, for $x, y \in \mathbb{R}^{n}$, by (10), we have

$$
J\left(y^{\prime}\right) \Omega\left(x, y^{\prime}\right)=\sum_{m=1}^{\infty} \sum_{s=1}^{D_{m}} a_{m, s}(x) Y_{m, s}\left(y^{\prime}\right),
$$

where

$$
a_{m, s}(x)=\int_{S^{n-1}} \Omega\left(x, y^{\prime}\right) Y_{m, s}\left(y^{\prime}\right) d \sigma\left(y^{\prime}\right) .
$$

So we get

$$
\Omega\left(x, y^{\prime}\right)=\sum_{m=1}^{\infty} \sum_{s=1}^{D_{m}} a_{m, s}(x) \frac{Y_{m, s}\left(y^{\prime}\right)}{J\left(y^{\prime}\right)} .
$$

In this way, (14) and (23) imply for any $x \in \mathbb{R}^{n}$

$$
\left|a_{m, s}(x)\right| \leq C(n, l) m^{-2 l},
$$

for any integer $l>1$. We fix hereafter $l=n$. Let

$$
\begin{aligned}
& g_{m, s}(f)(x) \\
& =\left\{\int_{0}^{\infty}\left|\int_{\rho(x-y) \leq t} \frac{Y_{m, s}\left((x-y)^{\prime}\right)}{J\left((x-y)^{\prime}\right) \rho(x-y)^{\alpha-1}} f(y) d y\right|^{2} \frac{d t}{t^{3}}\right\}^{1 / 2}, \\
& g_{m, s ; b} f(x) \\
& =\left\{\int_{0}^{\infty} \mid \int_{\rho(x-y) \leq t} \frac{Y_{m, s}\left((x-y)^{\prime}\right)}{J\left((x-y)^{\prime}\right) \rho(x-y)^{\alpha-1}}\right. \\
& \left.\times\left.(b(x)-b(y)) f(y) d y\right|^{2} \frac{d t}{t^{3}}\right\}^{1 / 2} .
\end{aligned}
$$

It is easy to see that $\Omega_{m, s}(x)=\left(Y_{m, s} / J\right)\left(x^{\prime}\right)$ has the following properties:

$$
\begin{gathered}
\int_{S^{n-1}} \Omega_{m, s}\left(x^{\prime}\right) J\left(x^{\prime}\right) d \sigma\left(x^{\prime}\right)=0, \\
\Omega_{m, s}\left(A_{\lambda} x\right)=\Omega_{m, s}(x), \quad \forall \lambda>0, x \in \mathbb{R}^{n} \backslash\{0\} .
\end{gathered}
$$

Moreover, $\Omega_{m, s} \in L^{2}\left(S^{n-1}\right) \quad \subset \quad L\left(\log ^{+} L\right)^{2}\left(S^{n-1}\right) \quad \subset$ $L \log ^{+} L\left(S^{n-1}\right)$. Then by the result in $[11,12]$, we know for $1<p<\infty$,

$$
\begin{gathered}
\left\|g_{m, s} f\right\|_{p} \leq C\|f\|_{p}, \\
\left\|g_{m, s ; b} f\right\|_{p} \leq C\|b\|_{*, \rho}\|f\|_{p},
\end{gathered}
$$

where $C$ is independent of $f, m, s$.
For any $z \in \mathbb{R}^{n}$, by the Minkowski inequality, the Hölder inequality, we get

$$
\begin{aligned}
& \left|g_{\Omega ; b} f(x)-g_{\Omega ; b} f(x+z)\right| \\
& \leq\left\{\int_{0}^{\infty} \mid \sum_{m=1}^{\infty} \sum_{s=1}^{D_{m}} a_{m, s}(x)\right.
\end{aligned}
$$

$$
\begin{aligned}
\times\left(\int_{\rho(x-y) \leq t} \frac{Y_{m, s}\left((x-y)^{\prime}\right)}{J\left((x-y)^{\prime}\right) \rho(x-y)^{\alpha-1}}\right. \\
\times(b(x)-b(y)) f(y) d y
\end{aligned}
$$$$
-\int_{\rho(x+z-y) \leq t}\left(\left(Y_{m, s}\left((x+z-y)^{\prime}\right)\right)\right.
$$

$$
\times\left(J\left((x+z-y)^{\prime}\right)\right.
$$

$$
\begin{array}{r}
\left.\left.\times \rho(x+z-y)^{\alpha-1}\right)^{-1}\right) \\
\left.\times(b(x+z)-b(y)) f(y) d y)\left.\right|^{2} \frac{d t}{t^{3}}\right\}^{1 / 2}
\end{array}
$$$$
+\left\{\int_{0}^{\infty} \mid \sum_{m=1}^{\infty} \sum_{s=1}^{D_{m}}\left(a_{m, s}(x)-a_{m, s}(x+z)\right)\right.
$$$$
\times \int_{\rho(x+z-y) \leq t} \frac{Y_{m, s}\left((x+z-y)^{\prime}\right)}{J\left((x+z-y)^{\prime}\right) \rho(x+z-y)^{\alpha-1}}
$$$$
\left.\times\left.(b(x+z)-b(y)) f(y) d y\right|^{2} \frac{d t}{t^{3}}\right\}^{1 / 2}
$$

$$
\begin{aligned}
= & \left\{\int_{0}^{\infty}\left|\sum_{m=1}^{\infty} \sum_{s=1}^{D_{m}} a_{m, s}(x) I_{m, s}(x, t, z)\right|^{2} \frac{d t}{t^{3}}\right\}^{1 / 2} \\
& +\left\{\int_{0}^{\infty} \mid \sum_{m=1}^{\infty} \sum_{s=1}^{D_{m}}\left(a_{m, s}(x)-a_{m, s}(x+z)\right)\right.
\end{aligned}
$$

$$
\begin{gathered}
\left.\times\left. I I_{m, s}(x, t, z)\right|^{2} \frac{d t}{t^{3}}\right\}^{1 / 2} \\
\leq\left\{\int_{0}^{\infty} \sum_{m=1}^{\infty} m^{-2} \sum_{m=1}^{\infty} m^{2} \mid \sum_{s=1}^{D_{m}} a_{m, s}(x) I_{m, s}\right. \\
+\left\{\int_{0}^{\infty} \sum_{m=1}^{\infty} m^{-2} \sum_{m=1}^{\infty} m^{2} \mid \sum_{s=1}^{D_{m}}\left(a_{m, s}(x)-a_{m, s}(x+z)\right)\right.
\end{gathered}
$$




$$
\begin{aligned}
\left.\times\left. I I_{m, s}(x, t, z)\right|^{2} \frac{d t}{t^{3}}\right\}^{1 / 2} & +C \sum_{m=1}^{\infty} m\left(\sum_{s=1}^{D_{m}}\left|a_{m, s}(x)-a_{m, s}(x+z)\right|^{2}\right)^{1 / 2} \\
\leq C\left\{\int_{0}^{\infty} \sum_{m=1}^{\infty} m^{2} \sum_{s=1}^{D_{m}}\left|a_{m, s}(x)\right|^{2}\right. & \times \sum_{s=1}^{D_{m}}\left(\int_{0}^{\infty}\left|I I_{m, s}(x, t, z)\right|^{2} \frac{d t}{t^{3}}\right)^{1 / 2} \\
& :=K_{1}+K_{2} .
\end{aligned}
$$$$
\left.\times \sum_{s=1}^{D_{m}}\left|I_{m, s}(x, t, z)\right|^{2} \frac{d t}{t^{3}}\right\}^{1 / 2}
$$$$
+C\left\{\int_{0}^{\infty} \sum_{m=1}^{\infty} m^{2} \sum_{s=1}^{D_{m}}\left|a_{m, s}(x)-a_{m, s}(x+z)\right|^{2}\right.
$$$$
\left.\times \sum_{s=1}^{D_{m}}\left|I I_{m, s}(x, t, z)\right|^{2} \frac{d t}{t^{3}}\right\}^{1 / 2},
$$

where

$$
\begin{aligned}
& I_{m, s}(x, t, z) \\
& =\left(\int_{\rho(x-y) \leq t} \frac{Y_{m, s}\left((x-y)^{\prime}\right)}{J\left((x-y)^{\prime}\right) \rho(x-y)^{\alpha-1}}\right. \\
& \quad \times(b(x)-b(y)) f(y) d y \\
& \quad-\int_{\rho(x+z-y) \leq t} \frac{Y_{m, s}\left((x+z-y)^{\prime}\right)}{J\left((x+z-y)^{\prime}\right) \rho(x+z-y)^{\alpha-1}} \\
& \quad \times(b(x+z)-b(y)) f(y) d y),
\end{aligned}
$$

$$
\begin{aligned}
& I I_{m, s}(x, t, z) \\
& =\int_{\rho(x+z-y) \leq t} \frac{Y_{m, s}\left((x+z-y)^{\prime}\right)}{J\left((x+z-y)^{\prime}\right) \rho(x+z-y)^{\alpha-1}} \\
& \quad \times(b(x+z)-b(y)) f(y) d y .
\end{aligned}
$$

Then by (43) and the Minkowski inequality, we get

$$
\begin{aligned}
& \left|g_{\Omega ; b} f(x)-g_{\Omega ; b} f(x+z)\right| \\
& \quad \leq C \sum_{m=1}^{\infty} m^{-3 n / 2} \sum_{s=1}^{D_{m}}\left(\int_{0}^{\infty}\left|I_{m, s}(x, t, z)\right|^{2} \frac{d t}{t^{3}}\right)^{1 / 2}
\end{aligned}
$$

We first give the $L^{p}$ norm of $K_{2}$. By (16), we have

$$
\left|a_{m, s}(x+z)-a_{m, s}(x)\right| \leq \mathrm{Cm}^{-2 n} \rho(z) .
$$

Moreover, by (47), we get

$$
\begin{aligned}
\left\|\left(\int_{0}^{\infty}\left|I I_{m, s}(\cdot, t, z)\right|^{2} \frac{d t}{t^{3}}\right)^{1 / 2}\right\|_{p} & =\left\|g_{m, s ; b} f(\cdot+z)\right\|_{L^{p}} \\
& \leq C\|f\|_{p} .
\end{aligned}
$$

Therefore, using $D_{m} \simeq m^{n-2}$ and the Minkowski inequality, we get

$$
\left\|K_{2}\right\|_{p} \leq C \sum_{m=1}^{\infty} m^{-2} \rho(z)\|f\|_{p} \leq C \rho(z)\|f\|_{p} .
$$

Now, we give the $L^{p}$ norm of $K_{1}$. Take an arbitrary $4^{-1}>\varepsilon^{2}>$ 0 and $z \in \mathbb{R}^{n}$ such that $\varepsilon^{-2} \rho(z) \leq 1$. Decompose $I_{m, s}$ as

$$
\begin{aligned}
& I_{m, s}(x, t, z) \\
& =\int_{\substack{\rho(x-y)>\varepsilon^{-2} \rho(z) \\
\rho(x-y) \leq t, \rho(x+z-y) \geq t}} \frac{Y_{m, s}\left((x-y)^{\prime}\right)}{J\left((x-y)^{\prime}\right) \rho(x-y)^{\alpha-1}} \\
& \times(b(x+z)-b(y)) f(y) d y \\
& +\int_{\substack{\rho(x-y)>\varepsilon^{-2} \rho(z) \\
\rho(x-y) \geq t, \rho(x+z-y) \leq t}} \frac{Y_{m, s}\left((x+z-y)^{\prime}\right)}{J\left((x+z-y)^{\prime}\right) \rho(x+z-y)^{\alpha-1}} \\
& \times(b(y)-b(x+z)) f(y) d y \\
& +\int_{\substack{\rho(x-y)>\varepsilon^{-2} \rho(z) \\
\rho(x-y) \leq t, \rho(x+z-y) \leq t}}\left(\frac{Y_{m, s}\left((x-y)^{\prime}\right)}{J\left((x-y)^{\prime}\right) \rho(x-y)^{\alpha-1}}\right. \\
& -\left(\left(Y_{m, s}\left((x+z-y)^{\prime}\right)\right)\right. \\
& \times\left(J\left((x+z-y)^{\prime}\right)\right. \\
& \left.\left.\left.\times \rho(x+z-y)^{\alpha-1}\right)^{-1}\right)\right) \\
& \times(b(x+z)-b(y)) f(y) d y \\
& +\int_{\substack{\rho(x-y)>\varepsilon^{-2} \rho(z) \\
\rho(x-y) \leq t}} \frac{Y_{m, s}\left((x-y)^{\prime}\right)}{J\left((x-y)^{\prime}\right) \rho(x-y)^{\alpha-1}}
\end{aligned}
$$




$$
\begin{gathered}
\times(b(x)-b(x+z)) f(y) d y \\
+\int_{\substack{\rho(x-y)>\varepsilon^{-2} \rho(z) \\
\rho(x-y) \leq t}} \frac{Y_{m, s}\left((x-y)^{\prime}\right)}{J\left((x-y)^{\prime}\right) \rho(x-y)^{\alpha-1}} \\
\times(b(x)-b(y)) f(y) d y \\
-\int_{\substack{\rho(x-y)>\varepsilon^{-2} \rho(z) \\
\rho(x+z-y) \leq t}} \frac{Y_{m, s}\left((x+z-y)^{\prime}\right)}{J\left((x+z-y)^{\prime}\right) \rho(x+z-y)^{\alpha-1}} \\
\quad \times(b(x+z)-b(y)) f(y) d y \\
=J_{1}(x, t)+J_{2}(x, t)+J_{3}(x, t)+J_{4}(x, t) \\
+J_{5}(x, t)-J_{6}(x, t) .
\end{gathered}
$$

We will estimate each term, respectively. For $J_{1}$, when $\rho(x-$ $y)>4 \rho(z)$, by Lemma $8,(21),|b(x+z)-b(y)|<C, J\left(x^{\prime}\right) \geq 1$, and the Minkowski inequality, we have

$$
\begin{aligned}
& \left\{\int_{0}^{\infty}\left|J_{1}(x, t)\right|^{2} \frac{d t}{t^{3}}\right\}^{1 / 2} \\
& \leq C \int_{\rho(x-y)>\varepsilon^{-2} \rho(z)} \frac{\left|Y_{m, s}\left((x-y)^{\prime}\right)\right|}{\rho(x-y)^{\alpha-1}}|f(y)| \\
& \quad \times\left\{\int_{\rho(x-y) \leq t, \rho(x+z-y) \geq t} \frac{d t}{t^{3}}\right\}^{1 / 2} d y \\
& \leq C \int_{\rho(x-y)>\varepsilon^{-2} \rho(z)} \frac{\left|Y_{m, s}\left((x-y)^{\prime}\right)\right|}{\rho(x-y)^{\alpha-1}}|f(y)| \\
& \leq C m^{n / 2-1} \int_{\rho(x-y)>\varepsilon^{-2} \rho(z)} \times\left\{\frac{1}{\rho(x-y)^{\alpha+1 / 2}}|f(y)| d y .\right.
\end{aligned}
$$

Then by the Minkowski inequality, we get

$$
\begin{aligned}
& \left\|\left\{\int_{0}^{\infty}\left|J_{1}(x, t)\right|^{2} \frac{d t}{t^{3}}\right\}^{1 / 2}\right\| \\
& \leq C m^{n / 2-1}\left\{\int _ { \mathbb { R } ^ { n } } \left(\int_{\rho(y)>\varepsilon^{-2} \rho(z)} \frac{\rho(z)^{1 / 2}}{\rho(y)^{\alpha+1 / 2}}\right.\right. \\
& \left.\times|f(x-y)| d y)^{p} d x\right\}^{1 / p} \\
& \leq C m^{n / 2-1}\|f\|_{p} \int_{\rho(y)>\varepsilon^{-2} \rho(z)} \frac{\rho(z)^{1 / 2}}{\rho(y)^{\alpha+1 / 2}} d y
\end{aligned}
$$

$$
\begin{aligned}
& \leq C m^{n / 2-1} \rho(z)^{1 / 2}\|f\|_{p} \int_{\mathcal{\varepsilon}^{-2} \rho(z)}^{\infty} \frac{d r}{r^{1+1 / 2}} \int_{S^{n-1}} d \sigma\left(y^{\prime}\right) \\
& \leq C m^{n / 2-1} \varepsilon\|f\|_{p} .
\end{aligned}
$$

Similar to the estimate of $J_{1}(x, t)$, we can get

$$
\begin{aligned}
& \left\{\int_{0}^{\infty}\left|J_{2}(x, t)\right|^{2} \frac{d t}{t^{3}}\right\}^{1 / 2} \\
& \leq C m^{n / 2-1} \int_{\rho(x-y)>\varepsilon^{-2} \rho(z)} \frac{\rho(z)^{1 / 2}}{\rho(x+z-y)^{\alpha+1 / 2}}|f(y)| d y .
\end{aligned}
$$

Then by the Minkowski inequality, we get

$$
\begin{aligned}
& \left\|\left\{\int_{0}^{\infty}\left|J_{2}(x, t)\right|^{2} \frac{d t}{t^{3}}\right\}^{1 / 2}\right\| \\
& \quad \leq C m^{n / 2-1} \\
& \quad \times\left\{\int _ { \mathbb { R } ^ { n } } \left(\int_{\rho(y)>\left(\varepsilon^{-2}-1\right) \rho(z)} \frac{\rho(z)^{1 / 2}}{\rho(y)^{\alpha+1 / 2}}\right.\right. \\
& \left.\quad \times|f(x+z-y)| d y)^{p} d x\right\}^{1 / p} \\
& \leq C m^{n / 2-1}\|f\|_{p} \rho(z)^{1 / 2} \int_{\rho(y)>\left(\varepsilon^{-2}-1\right) \rho(z)} \frac{1}{\rho(y)^{\alpha+1 / 2}} d y \\
& \leq C m^{n / 2-1}\left(\varepsilon^{-2}-1\right)^{-1 / 2}\|f\|_{p} \\
& \leq C m^{n / 2-1} \varepsilon\|f\|_{p} \cdot
\end{aligned}
$$

For $J_{3}$, since $|b(x+z)-b(y)|<C$, by Lemma 6 , the Minkowski inequality, and Lemma 8 , we have

$$
\begin{aligned}
& \left\{\int_{0}^{\infty}\left|J_{3}(x, t)\right|^{2} \frac{d t}{t^{3}}\right\}^{1 / 2} \\
& \leq C \int_{\rho(x-y)>\varepsilon^{-2} \rho(z)} \mid \frac{Y_{m, s}\left((x-y)^{\prime}\right)}{J\left((x-y)^{\prime}\right) \rho(x-y)^{\alpha-1}} \\
& \quad-\frac{Y_{m, s}\left((x+z-y)^{\prime}\right)}{J\left((x+z-y)^{\prime}\right) \rho(x+z-y)^{\alpha-1}} \mid \\
& \quad \times|f(y)|\left\{\int_{\rho(x-y) \leq t} \frac{d t}{t^{3}}\right\}^{1 / 2} d y \\
& \leq C m^{n / 2} \int_{\rho(x-y)>\varepsilon^{-2} \rho(z)} \frac{\rho(z)}{\rho(x-y)^{\alpha+1}} d y .
\end{aligned}
$$


Then similar to estimate (56), we get

$$
\left\|\left\{\int_{0}^{\infty}\left|J_{3}(x, t)\right|^{2} \frac{d t}{t^{3}}\right\}^{1 / 2}\right\|_{p} \leq C m^{n / 2} \varepsilon\|f\|_{p} .
$$

About $J_{4}$, we have

$$
\begin{aligned}
& \left(\int_{0}^{\infty}\left|J_{4}(x, t)\right|^{2} \frac{d t}{t^{3}}\right)^{1 / 2} \\
& \leq|(b(x)-b(x+z))| \\
& \quad \times\left\{\int_{0}^{\infty} \mid \int_{\begin{array}{c}
\rho(x-y)>\varepsilon^{-2} \rho(z) \\
\rho(x-y) \leq t
\end{array}} \frac{Y_{m, s}\left((x-y)^{\prime}\right)}{J\left((x-y)^{\prime}\right) \rho(x-y)^{\alpha-1}}\right. \\
& \left.\quad \times\left. f(y) d y\right|^{2} \frac{d t}{t^{3}}\right\}^{1 / 2} \\
& =|(b(x)-b(x+z))| g_{m, s}^{\varepsilon^{-2} \rho(z)} f(x) .
\end{aligned}
$$

Similar to the estimate of $S_{2^{1 / \varepsilon}|v|}^{\rho} f(x)$ in [35], we get for any fixed $1<q<p<\infty$,

$$
g_{m, s}^{\varepsilon^{-2} \rho(z)} f(x) \leq C \mathscr{M}\left(g_{m, s} f\right)(x)+C\left(\mathscr{M}|f|^{q}(x)\right)^{1 / q},
$$

where $C$ is independent of $m, s, \varepsilon$ and $\rho(z)$. So, for any $1<$ $p<\infty$, taking $1<q<p$ in (62), then by (43) and the $L^{p}$ boundedness of $\left(\mathscr{M}\left(|f|^{q}\right)\right)^{1 / q}$, we get

$$
\left\|g_{m, s}^{\varepsilon^{-2} \rho(z)} f\right\|_{p} \leq C\|f\|_{p} .
$$

Since $b \in C_{0}^{\infty}$, we have $|b(x)-b(x+z)| \leq C \rho(z)$. Then by (63), we get

$$
\left\|\left(\int_{0}^{\infty}\left|J_{4}(x, t)\right|^{2} \frac{d t}{t^{3}}\right)^{1 / 2}\right\|_{p} \leq C m^{n / 2} \rho(z)\|f\|_{p} .
$$

For $J_{5}$, since $J\left(x^{\prime}\right) \geq 1$, and $|b(x)-b(y)| \leq C \rho(x-y)$ when $\rho(x-y) \leq 1$, then by (21) and the Minkowski inequality, we get

$$
\begin{aligned}
& \left\{\int_{0}^{\infty}\left|J_{5}(x, t)\right|^{2} \frac{d t}{t^{3}}\right\}^{1 / 2} \\
& \quad \leq C m^{n / 2-1} \int_{\rho(x-y) \leq \varepsilon^{-2} \rho(z)} \frac{|f(y)|}{\rho(x-y)^{\alpha-1}} d y .
\end{aligned}
$$

Then we can get

$$
\left\|\left\{\int_{0}^{\infty}\left|J_{5}(x, t)\right|^{2} \frac{d t}{t^{3}}\right\}^{1 / 2}\right\|_{p} \leq C m^{n / 2-1} \varepsilon^{-2} \rho(z) .
$$

Similarly, we can get

$$
\left\|\left\{\int_{0}^{\infty}\left|J_{6}(x, t)\right|^{2} \frac{d t}{t^{3}}\right\}^{1 / 2}\right\|_{p} \leq C D m^{n / 2-1}\left(\varepsilon^{-2} \rho(z)+\rho(z)\right) .
$$

Combining the estimate of $J_{1}$ with $J_{6}$, by the Minkowski inequality and $D_{m} \simeq C m^{(n-2)}$, we get

$$
\begin{aligned}
\left\|K_{1}\right\|_{p} & \leq C \sum_{m=1}^{\infty} m^{-3 n / 2} \sum_{s=1}^{D_{m}}\left\|\left(\int_{0}^{\infty}\left|I_{m, s}(x, t)\right|^{2} \frac{d t}{t^{3}}\right)^{1 / 2}\right\|_{p} \\
& \leq C \sum_{m=1}^{\infty} m^{-3 n / 2} \sum_{s=1}^{D_{m}} m^{n / 2-1}\left(\varepsilon^{-2} \rho(z)+\rho(z)+\varepsilon\right)\|f\|_{p} \\
& \leq C \sum_{m=1}^{\infty} m^{-3 n / 2} m^{n-2+n / 2-1}\left(\varepsilon^{-2} \rho(z)+\rho(z)+\varepsilon\right)\|f\|_{p} \\
& \leq C \sum_{m=1}^{\infty} m^{-3}\left(\varepsilon^{-2} \rho(z)+\rho(z)+\varepsilon\right)\|f\|_{p} \\
& \leq C\left(\varepsilon^{-2} \rho(z)+\rho(z)+\varepsilon\right)\|f\|_{p},
\end{aligned}
$$

which combined the $L^{p}$ norm of $K_{2}$ (see (53)); then by taking $\rho(z)$ sufficiently small depending on $\varepsilon$, we can get

$$
\left\|g_{\Omega ; b} f(\cdot)-g_{\Omega ; b} f(\cdot+z)\right\|_{p} \leq C \varepsilon
$$

Thus we establish the proof of Theorem 2.

\section{Proof of Theorem 3}

In this proof for $j=1,2, \ldots, 13, B_{j}$ is a positive constant depending only on $\Omega, p, n, \delta, \Theta$ and $B_{i}(1 \leq i<j)$. Suppose that $g_{\Omega ; b}$ is a bounded operator from $L^{p}\left(\mathbb{R}^{n}\right)$ to $L^{p}\left(\mathbb{R}^{n}\right)$; we are going to prove that $b \in \mathrm{BMO}$. Without loss of generality, we can assume $\left\|g_{\Omega ; b}\right\|_{L^{p \mapsto L^{p}}}=1$. To prove that $b \in \operatorname{BMO}\left(\mathbb{R}^{n}, \rho\right)$, we need to show there exists a constant $B>0$ such that for any $x_{0} \in \mathbb{R}^{n}$ and $r>0$,

$$
\begin{aligned}
N & =\frac{1}{\left|\mathscr{E}\left(x_{0}, r\right)\right|} \int_{\mathscr{E}\left(x_{0}, r\right)}\left|b(y)-b_{\mathscr{E}\left(x_{0}, r\right)}\right| d y \\
& \leq B(p, \Omega, \Theta, \delta, n),
\end{aligned}
$$

where $b_{\mathscr{E}\left(x_{0}, r\right)}=\left(1 /\left|\mathscr{E}\left(x_{0}, r\right)\right|\right) \int_{\mathscr{E}\left(x_{0}, r\right)} b(y) d y$. Since $g_{\Omega ; b-b_{\mathscr{E}\left(x_{0}, r\right)}}=g_{\Omega ; b}$, we may assume $b_{\mathscr{E}\left(x_{0}, r\right)}=0$. So it suffices to prove that for any fixed $x_{0} \in \mathbb{R}^{n}, r \in \mathbb{R}_{+}$,

$$
N=\frac{1}{\left|\mathscr{E}\left(x_{0}, r\right)\right|} \int_{\mathscr{E}\left(x_{0}, r\right)}|b(y)| d y \leq B(p, \Omega, \Theta, \delta, n),
$$

where $\left(1 /\left|\mathscr{E}\left(x_{0}, r\right)\right|\right) \int_{\mathscr{E}\left(x_{0}, r\right)} b(y) d y=0$. Let

$$
f(y)=\left[\operatorname{sgn}(b(y))-c_{0}\right] \chi_{\mathscr{E}\left(x_{0}, r\right)}(y)
$$


where $c_{0}=\left|\mathscr{E}\left(x_{0}, r\right)\right|^{-1} \int_{\mathscr{E}\left(x_{0}, r\right)} \operatorname{sgn}(b(y)) d y$. We get $\left|c_{0}\right|<1$. Then $f$ satisfies the following properties:

$$
\begin{gathered}
\|f\|_{\infty} \leq 2, \\
\operatorname{supp} f \subset \mathscr{E}\left(x_{0}, r\right), \\
\int_{\mathbb{R}^{n}} f(y) d y=0, \\
f(y) b(y)>0, \\
\frac{1}{\left|\mathscr{E}\left(x_{0}, r\right)\right|} \int_{\mathbb{R}^{n}} f(y) b(y) d y=N .
\end{gathered}
$$

Then for $x \in G=\left\{x \in \mathbb{R}^{n}: \rho\left(x-x_{0}\right)>B_{1} r=\left(3 \delta^{-1}+1\right) r\right.$ and $\left.\left(x-x_{0}\right)^{\prime} \in \Theta\right\}$,

$$
\begin{aligned}
& \left|g_{\Omega ; b} f(x)\right| \\
& \geq\left|g_{\Omega}(b f)(x)\right|-|b(x)|\left|g_{\Omega} f(x)\right| \\
& =\left(\int_{0}^{\infty} \mid \int_{\rho(x-y) \leq t} \Omega\left(x,(x-y)^{\prime}\right)\right. \\
& \left.\quad \times\left.\rho(x-y)^{1-\alpha} b(y) f(y) d y\right|^{2} \frac{d t}{t^{3}}\right)^{1 / 2} \\
& -|b(x)|\left(\int_{0}^{\infty} \mid \int_{\rho(x-y) \leq t} \Omega\left(x,(x-y)^{\prime}\right)\right. \\
& \left.\quad \times\left.\rho(x-y)^{1-\alpha} f(y) d y\right|^{2} \frac{d t}{t^{3}}\right)^{1 / 2} \\
& :=I_{1}-I_{2} .
\end{aligned}
$$

For $I_{1}$, by (18), we know

$$
\Omega\left(x,\left(x-x_{0}\right)^{\prime}\right) \geq 2 \delta .
$$

Noting that if $\rho\left(y-x_{0}\right)<r$ and $\rho\left(x-x_{0}\right)>B_{1} r$, by Lemma 9 , we get $\rho\left((x-y)^{\prime}-\left(x-x_{0}\right)^{\prime}\right) \leq \delta$. Since

$$
\begin{gathered}
\sup _{x \in \mathbb{R}^{n}}\left|\Omega\left(x,(x-y)^{\prime}\right)-\Omega\left(x,\left(x-x_{0}\right)^{\prime}\right)\right| \\
\leq \rho\left((x-y)^{\prime}-\left(x-x_{0}\right)^{\prime}\right) \leq \delta,
\end{gathered}
$$

if $\Omega\left(x,\left(x-x_{0}\right)^{\prime}\right) \geq 2 \delta$, we can get $\Omega\left(x,(x-y)^{\prime}\right) \geq \delta$. Then by (74), (76), (77), (3/4) $\rho\left(x-x_{0}\right) \leq \rho(x-y) \leq(5 / 4) \rho\left(x-x_{0}\right)$ and the Hölder inequality, we get

$$
\begin{aligned}
I_{1} \geq & \delta\left\{\int _ { \rho ( x - x _ { 0 } ) } ^ { \infty } \left(\int_{\mathscr{E}\left(x_{0}, r\right)} b(y) f(y) \rho(x-y)^{1-\alpha}\right.\right. \\
& \left.\left.\times \chi_{\{\rho(x-y) \leq t\}} d y\right)^{2} \frac{d t}{t^{3}}\right\}^{1 / 2} \\
\geq & \delta \int_{\rho\left(x-x_{0}\right)}^{\infty} \int_{\mathscr{E}\left(x_{0}, r\right)} b(y) f(y) \rho(x-y)^{1-\alpha} \chi_{\{\rho(x-y) \leq t\}} d y \frac{d t}{t^{3}} \\
& \times\left(\int_{\rho\left(x-x_{0}\right)}^{\infty} \frac{d t}{t^{3}}\right)^{-1 / 2} \\
= & C \delta \rho\left(x-x_{0}\right) \int_{\mathscr{E}\left(x_{0}, r\right)} \rho(x-y)^{1-\alpha} b(y) f(y) \\
\geq & B_{2} \delta \rho\left(x-x_{0}\right)^{-\alpha} \int_{\mathscr{E}\left(x_{0}, r\right)} b(y) f(y) d y \\
= & B_{3} N r^{\alpha} \rho\left(x-x_{0}\right)^{-\alpha} .
\end{aligned}
$$

By (73), (74), (75), $\rho\left(x-x_{0}\right) \simeq \rho(x-y), \rho\left(y-x_{0}\right)<r$ and $\Omega \in L^{\infty}\left(\mathbb{R}^{n}\right) \times L^{\infty}\left(S^{n-1}\right)$, then by Lemma 13 and Lemma 8 , we have

$$
\begin{aligned}
& I_{2} \\
& =|b(x)| \\
& \times\left(\int_{0}^{\infty} \mid \int_{\mathbb{R}^{n}}\left(\frac{\Omega(x, x-y)}{\rho(x-y)^{\alpha-1}} \chi_{\{\rho(x-y) \leq t\}}\right.\right. \\
& \left.-\frac{\Omega\left(x, x-x_{0}\right)}{\rho\left(x-x_{0}\right)^{\alpha-1}} \chi_{\left\{\rho\left(x-x_{0}\right) \leq t\right\}}\right) \\
& \left.\times\left. f(y) d y\right|^{2} \frac{d t}{t^{3}}\right)^{1 / 2} \\
& \leq|b(x)| \int_{\mathscr{E}\left(x_{0}, r\right)}|f(y)|\left|\frac{\Omega(x, x-y)}{\rho(x-y)^{\alpha-1}}-\frac{\Omega\left(x, x-x_{0}\right)}{\rho\left(x-x_{0}\right)^{\alpha-1}}\right| \\
& \times\left(\int_{\substack{\rho(x-y) \leq t \\
\rho\left(x-x_{0}\right) \leq t}} \frac{d t}{t^{3}}\right)^{1 / 2} d y \\
& +|b(x)| \int_{\mathscr{E}\left(x_{0}, r\right)} \frac{|\Omega(x, x-y)|}{\rho(x-y)^{\alpha-1}}|f(y)| \\
& \times\left(\int_{\substack{\rho(x-y) \leq t \\
\rho\left(x-x_{0}\right) \geq t}} \frac{d t}{t^{3}}\right)^{1 / 2} d y
\end{aligned}
$$




$$
\begin{aligned}
& +|b(x)| \int_{\mathscr{E}\left(x_{0}, r\right)} \frac{\left|\Omega\left(x, x-x_{0}\right)\right|}{\rho\left(x-x_{0}\right)^{\alpha-1}}|f(y)| \\
& \quad \times\left(\int_{\substack{\rho(x-y) \geq t \\
\rho\left(x-x_{0}\right) \leq t}} \frac{d t}{t^{3}}\right)^{1 / 2} d y \\
& \leq C|b(x)| r \int_{\mathscr{C}\left(x_{0}, r\right)} \frac{|f(y)|}{\rho\left(x-x_{0}\right)^{\alpha+1}} d y \\
& +C|b(x)| r^{1 / 2} \int_{\mathscr{E}\left(x_{0}, r\right)} \frac{|f(y)|}{\rho\left(x-x_{0}\right)^{\alpha+1 / 2}} d y \\
& \leq B_{4}|b(x)| r^{\alpha+1 / 2} \rho\left(x-x_{0}\right)^{-\alpha-1 / 2} .
\end{aligned}
$$

Then using the same argument of the proof of Theorem 1.2 in [29], we get $N \leq B(\Omega, n, \delta, \Theta, p)$.

\section{Proof of Theorem 4}

Since $g_{\Omega ; b}$ is a compact operator on $L^{p}\left(\mathbb{R}^{n}\right)$, then $g_{\Omega ; b}$ is bounded on $L^{p}\left(\mathbb{R}^{n}\right)$. Thus from Theorem $3, b \in \operatorname{BMO}\left(\mathbb{R}^{n}, \rho\right)$. Thus we may assume $\|b\|_{*, \rho}=1$. By Lemma 12 , to prove that $b \in \mathrm{CMO}\left(\mathbb{R}^{n}, \rho\right)$, it suffices to show that $b$ satisfies conditions (i), (ii), and (iii) of Lemma 12.

First, suppose that $b$ does not satisfy (i) of Lemma 12. Then there exists $\zeta>0$ and a sequence of ellipsoids $\left\{\mathscr{E}_{j}\left(y_{j}, r_{j}\right)\right\}_{j=1}^{\infty}$ with $\lim _{j \rightarrow \infty} r_{j}=0$, such that for every $j$

$$
M\left(b, \mathscr{E}_{j}\right)=\left|\mathscr{E}_{j}\right|^{-1} \int_{\mathscr{C}_{j}}\left|b(y)-b_{\mathscr{C}_{j}}\right| d y>\zeta .
$$

Let $f_{j}(y)=\left|\mathscr{E}_{j}\right|^{-1 / p}\left\{\left[\operatorname{sgn}\left(b(y)-b_{\mathscr{C}_{j}}\right)-c_{0}\right] \chi_{\mathscr{E}_{j}}(y)\right\}$, where $c_{0}=\left|\mathscr{E}_{j}\right|^{-1} \int_{\mathscr{C}_{j}} \operatorname{sgn}\left(b(y)-b_{\mathscr{C}_{j}}\right) d y$. So $\left|c_{0}\right|<1$. Then $f_{j}$ has the following properties:

$$
\begin{gathered}
\operatorname{supp} f_{j} \subset \mathscr{E}_{j} ; \\
f_{j}(y)\left(b(y)-b_{\mathscr{C}_{j}}\right)>0 \\
\int_{\mathbb{R}^{n}} f_{j}(y) d y=0 \\
\left|f_{j}(y)\right| \leq 2\left|\mathscr{E}_{j}\right|^{-1 / p}
\end{gathered}
$$

for $y \in \mathscr{E}_{j}$. It is easy to see that $\left\|f_{j}\right\|_{p} \leq C$, where $C$ is independent of $j$.

Let $B_{1}=(3 / \delta)+1$. If $\rho\left(y-y_{j}\right)<r_{j}$, then

$$
x \in\left(B_{1} \mathscr{E}_{j}\right)^{c} \cap\left\{x:\left(x-y_{j}\right)^{\prime} \in \Theta\right\} .
$$

Similar to the proof of $I_{1}$ in Theorem 3, by (84), (85) and noting that $\rho\left(x-y_{j}\right) \simeq \rho(x-y)$, we get

$$
\begin{aligned}
& \left|g_{\Omega}\left(\left(b-b_{\mathscr{C}_{j}}\right) f_{j}\right)(x)\right| \\
& \geq \delta\left\{\int_{0}^{\infty}\left(\int_{\mathscr{C}_{j}} \frac{\left(b(y)-b_{\mathscr{C}_{j}}\right) f_{j}(y)}{\rho(x-y)^{\alpha-1}} \chi_{\{\rho(x-y) \leq t\}} d y\right)^{2} \frac{d t}{t^{3}}\right\}^{1 / 2} \\
& \geq \delta \int_{\rho\left(x-y_{j}\right)}^{\infty} \int_{\mathscr{C}_{j}} \frac{\left(b(y)-b_{\mathscr{C}_{j}}\right) f_{j}(y)}{\rho(x-y)^{\alpha-1}} \chi_{\{\rho(x-y) \leq t\}} d y \frac{d t}{t^{3}} \\
& \times\left(\int_{\rho\left(x-y_{j}\right)}^{\infty} \frac{d t}{t^{3}}\right)^{-1 / 2} \\
& \geq \delta\left|\mathscr{E}_{j}\right|^{-1 / p} \rho\left(x-y_{j}\right) \\
& \times \int_{\rho\left(x-y_{j}\right)}^{\infty} \int_{\mathscr{C}_{j}}\left(\left(\left(b(y)-b_{\mathscr{C}_{j}}\right)\right.\right. \\
& \left.\times\left\{\left[\operatorname{sgn}\left(b(y)-b_{\mathscr{C}_{j}}\right)-c_{0}\right] \chi_{\mathscr{E}_{j}}(y)\right\}\right) \\
& \left.\times\left(\rho(x-y)^{\alpha-1}\right)^{-1}\right) \\
& \times \chi_{\{\rho(x-y) \leq t\}} d y \frac{d t}{t^{3}} \\
& \geq \delta\left|\mathscr{E}_{j}\right|^{-1 / p} \rho\left(x-y_{j}\right)^{-\alpha} \\
& \times \int_{\mathscr{E}_{j}}\left|b(y)-b_{\mathscr{C}_{j}}\right|-c_{0}\left(b(y)-b_{\mathscr{C}_{j}}\right) d y \\
& =\delta\left|\mathscr{E}_{j}\right|^{-1 / p} \rho\left(x-y_{j}\right)^{-\alpha} \int_{\mathscr{E}_{j}}\left|b(y)-b_{\mathscr{C}_{j}}\right| d y \\
& \geq B_{2}\left|\mathscr{E}_{j}\right|^{-1 / p^{\prime}} \rho\left(x-y_{j}\right)^{-\alpha} .
\end{aligned}
$$

On the other hand, for $x \in\left(B_{1} \mathscr{E}_{j}\right)^{c}$, by (84), (87), $\Omega \in$ $L^{\infty}\left(\mathbb{R}^{n}\right) \times L^{\infty}\left(S^{n-1}\right)$, and $\rho\left(x-y_{j}\right) \simeq \rho(x-y)$ when $y \in \mathscr{E}_{j}$, we have

$$
\begin{aligned}
& \left|g_{\Omega}\left(\left(b-b_{\mathscr{C}_{j}}\right) f_{j}\right)(x)\right| \\
& \leq \int_{\mathscr{C}_{j}}\left|b(y)-b_{\mathscr{C}_{j}}\right|\left|f_{j}(y)\right| \frac{|\Omega(x, x-y)|}{\rho(x-y)^{\alpha-1}} \\
& \quad \times\left(\int_{\rho(x-y) \leq t} \frac{d t}{t^{3}}\right)^{1 / 2} d y \\
& \leq C\left|\mathscr{E}_{j}\right|^{1 / p^{\prime}} \rho\left(x-y_{j}\right)^{-\alpha} .
\end{aligned}
$$


On the other hand, for $x \in\left(B_{1} \mathscr{E}_{j}\right)^{c}$, by (84), (86), (87), and $\rho\left(x-y_{j}\right) \simeq \rho(x-y)$, we have

$$
\begin{aligned}
& \left|\left(b(x)-b_{\mathscr{C}_{j}}\right) g_{\Omega}\left(f_{j}\right)(x)\right| \\
& \leq\left|b(x)-b_{\mathscr{C}_{j}}\right| \\
& \times\left(\int_{0}^{\infty} \mid \int_{\substack{\rho(x-y) \leq t \\
\rho\left(x-y_{j}\right) \leq t}}\left(\frac{\Omega(x, x-y)}{\rho(x-y)^{\alpha-1}}-\frac{\Omega\left(x, x-y_{j}\right)}{\rho\left(x-y_{j}\right)^{\alpha-1}}\right)\right. \\
& \left.\times\left. f_{j}(y) d y\right|^{2} \frac{d t}{t^{3}}\right)^{1 / 2} \\
& +\left|b(x)-b_{\mathscr{C}_{j}}\right| \\
& \times\left(\int_{0}^{\infty}\left|\int_{\substack{\rho(x-y) \leq t \\
\rho\left(x-y_{j}\right)>t}} \frac{\Omega(x, x-y)}{\rho(x-y)^{\alpha-1}} f_{j}(y) d y\right|^{2} \frac{d t}{t^{3}}\right)^{1 / 2} \\
& +\left|b(x)-b_{\mathscr{C}_{j}}\right| \\
& \times\left(\int_{0}^{\infty}\left|\int_{\substack{\rho(x-y)>t \\
\rho\left(x-y_{j}\right) \leq t}} \frac{\Omega\left(x, x-y_{j}\right)}{\rho\left(x-y_{j}\right)^{\alpha-1}} f_{j}(y) d y\right|^{2} \frac{d t}{t^{3}}\right)^{1 / 2} \\
& \leq C\left|\mathscr{E}_{j}\right|^{1 / p^{\prime}} \frac{\left|b(x)-b_{\mathscr{C}_{j}}\right| r_{j}^{1 / 2}}{\rho\left(x-y_{j}\right)^{\alpha+1 / 2}}
\end{aligned}
$$

In fact, in the above estimate we have used Lemma 13 for $x \in$ $\left(B_{1} \mathscr{E}_{j}\right)^{c}$. Then similar to the proof of Theorem 1.3 in [29], we show that $b$ satisfies (i)-(iii) of Lemma 12 .

\section{Conflict of Interests}

The authors declare that there is no conflict of interests regarding the publication of this paper.

\section{Acknowledgments}

Y. Chen is supported by NSF of China (Grant no. 11471033), NCET of China (Grant no. NCET-11-0574), and the Fundamental Research Funds for the Central Universities (FRF-TP12-006B). Y. Ding is supported by NSF of China (Grant no. 11371057), SRFDP of China (Grant no. 20130003110003).

\section{References}

[1] R. R. Coifman, R. Rochberg, and G. Weiss, "Factorization theorems for Hardy spaces in several variables," Annals of Mathematics, vol. 103, no. 3, pp. 611-635, 1976.

[2] A. Uchiyama, "On the compactness of operators of Hankel type," Tohoku Mathematical Journal, vol. 30, no. 1, pp. 163-171, 1978.
[3] S. Janson, "Mean oscillation and commutators of singular integral operators," Arkiv för Matematik, vol. 16, no. 2, pp. 263270, 1978.

[4] F. Beatrous and S.-Y. Li, "On the boundedness and compactness of operators of Hankel type," Journal of Functional Analysis, vol. 111, no. 2, pp. 350-379, 1993.

[5] S. G. Krantz and S.-Y. Li, "Boundedness and compactness of integral operators on spaces of homogeneous type and applications. II," Journal of Mathematical Analysis and Applications, vol. 258, no. 2, pp. 642-657, 2001.

[6] E. B. Fabes and N. M. Rivière, "Singular integrals with mixed homogeneity," Studia Mathematica, vol. 27, pp. 19-38, 1966.

[7] W. R. Madych, "On Littlewood-Paley functions," Studia Mathematica, vol. 50, pp. 43-63, 1974.

[8] Q. Y. Xue, Y. Ding, and K. Yabuta, "Parabolic LittlewoodPaley $g$-function with rough kernel," Acta Mathematica Sinica, English Series, vol. 24, no. 12, pp. 2049-2060, 2008.

[9] A. Al-Salman, "Parabolic Marcinkiewicz integrals along surfaces on product domains," Acta Mathematica Sinica (English Series), vol. 27, no. 1, pp. 1-18, 2011.

[10] D. X. Chen and S. Z. Lu, " $L^{p}$ boundedness of the parabolic Littlewood-Paley operator with rough kernel belonging to $F\left(S^{n-1}\right)$," Acta Mathematica Scientia A, vol. 31, no. 2, pp. 343350, 2011.

[11] D. Chen and S. Lu, " $L^{p}$ boundedness for commutators of parabolic Littlewood-Paley operators with rough kernels," Mathematische Nachrichten, vol. 284, no. 8-9, pp. 973-986, 2011.

[12] Y. Chen and Y. Ding, "The parabolic Littlewood-Paley operator with Hardy space kernels," Canadian Mathematical Bulletin, vol. 52, no. 4, pp. 521-534, 2009.

[13] A. Al-Salman, H. Al-Qassem, L. C. Cheng, and Y. Pan, " $L^{p}$ bounds for the function of Marcinkiewicz," Mathematical Research Letters, vol. 9, no. 5-6, pp. 697-700, 2002.

[14] A. Benedek, A.-P. Calderón, and R. Panzone, "Convolution operators on Banach space valued functions," Proceedings of the National Academy of Sciences of the United States of America, vol. 48, pp. 356-365, 1962.

[15] J. Chen, D. Fan, and Y. Pan, "A note on a Marcinkiewicz integral operator," Mathematische Nachrichten, vol. 227, pp. 33-42, 2001.

[16] Y. Ding, D. Fan, and Y. Pan, "Weighted boundedness for a class of rough Marcinkiewicz integrals," Indiana University Mathematics Journal, vol. 48, no. 3, pp. 1037-1056, 1999.

[17] Y. Ding, D. Fan, and Y. Pan, " $L^{p}$-boundedness of Marcinkiewicz integrals with Hardy space function kernels," Acta Mathematica Sinica. English Series, vol. 16, no. 4, pp. 593-600, 2000.

[18] Y. Ding, S. Lu, and K. Yabuta, "On commutators of Marcinkiewicz integrals with rough kernel," Journal of Mathematical Analysis and Applications, vol. 275, no. 1, pp. 60-68, 2002.

[19] L. Hõrmander, "Estimates for translation invariant operators in $L^{p}$ spaces," Acta Mathematica, vol. 104, pp. 93-140, 1960.

[20] G. Hu and D. Yan, "On the commutator of the Marcinkiewicz integral," Journal of Mathematical Analysis and Applications, vol. 283, no. 2, pp. 351-361, 2003.

[21] E. M. Stein, "On the functions of Littlewood-Paley, LUSin, and Marcinkiewicz," Transactions of the American Mathematical Society, vol. 88, pp. 430-466, 1958.

[22] T. Walsh, "On the function of Marcinkiewicz," Studia Mathematica, vol. 44, pp. 203-217, 1972.

[23] H. Wu, "On Marcinkiewicz integral operators with rough kernels," Integral Equations and Operator Theory, vol. 52, no. 2, pp. 285-298, 2005. 
[24] D. K. Palagachev and L. G. Softova, "Singular integral operators, Morrey spaces and fine regularity of solutions to PDE's," Potential Analysis, vol. 20, no. 3, pp. 237-263, 2004.

[25] Y. Chen and Y. Ding, "Boundedness of commutators of Marcinkiewicz integral with rough variable kernel," Integral Equations and Operator Theory, vol. 61, no. 4, pp. 477-492, 2008.

[26] Y. Chen and D. Yong, " $L^{p}$ boundedness for Littlewood-Paley operators with rough variable kernels," Journal of Mathematical Analysis and Applications, vol. 377, no. 2, pp. 889-904, 2011.

[27] Y. Ding, C.-C. Lin, and S. Shao, "On the Marcinkiewicz integral with variable kernels," Indiana University Mathematics Journal, vol. 53, no. 3, pp. 805-821, 2004.

[28] Y. Ding and R. Li, "An integral estimate of Bessel function and its application," Science in China A: Mathematics, vol. 51, no. 5, pp. 897-906, 2008.

[29] Y. Chen and Y. Ding, "Compactness of commutators of singular inte grals with variable Kernels," Chinese Annals of Mathematics $A$, vol. 30, pp. 201-212, 2009 (Chinese).

[30] M. S. Berger, Nonlinearity and Functional Ananysis, Academic Press, New York, NY, USA, 1977.

[31] A. Calderón and A. Zygmund, "Singular integral operators and differential equations," American Journal of Mathematics, vol. 79, pp. 901-921, 1957.

[32] Y. Chen and Y. Ding, "Compactness of the commutators of parabolic singular integrals," Science China. Mathematics, vol. 53, no. 10, pp. 2633-2648, 2010.

[33] K. Yosida, Functional Analysis, Springer, New York, NY, USA, 1968.

[34] E. M. Stein and G. Weiss, Introdution to Fourier Analysis on Euclidean Spaces, Princeton University Press, Princeton, NJ, USA, 1971.

[35] Y. Chen and Y. Ding, "Compactness characterization of commutators for Littlewood-Paley operators," Kodai Mathematical Journal, vol. 32, no. 2, pp. 256-323, 2009. 


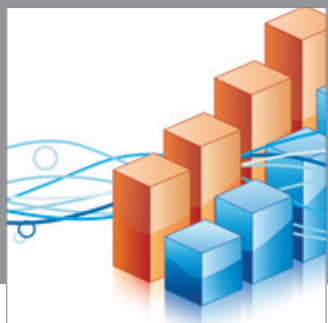

Advances in

Operations Research

mansans

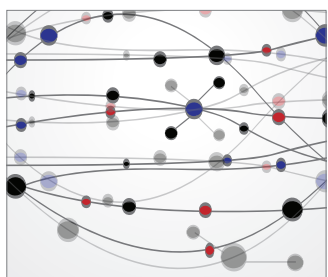

The Scientific World Journal
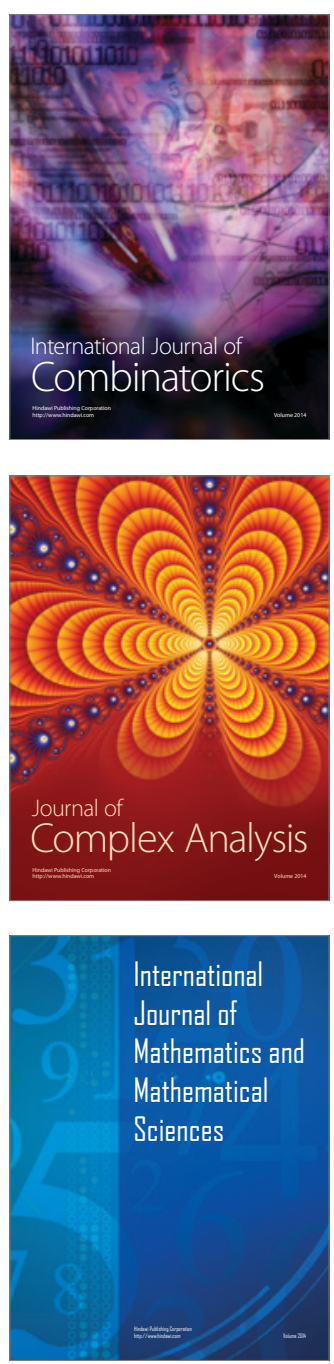
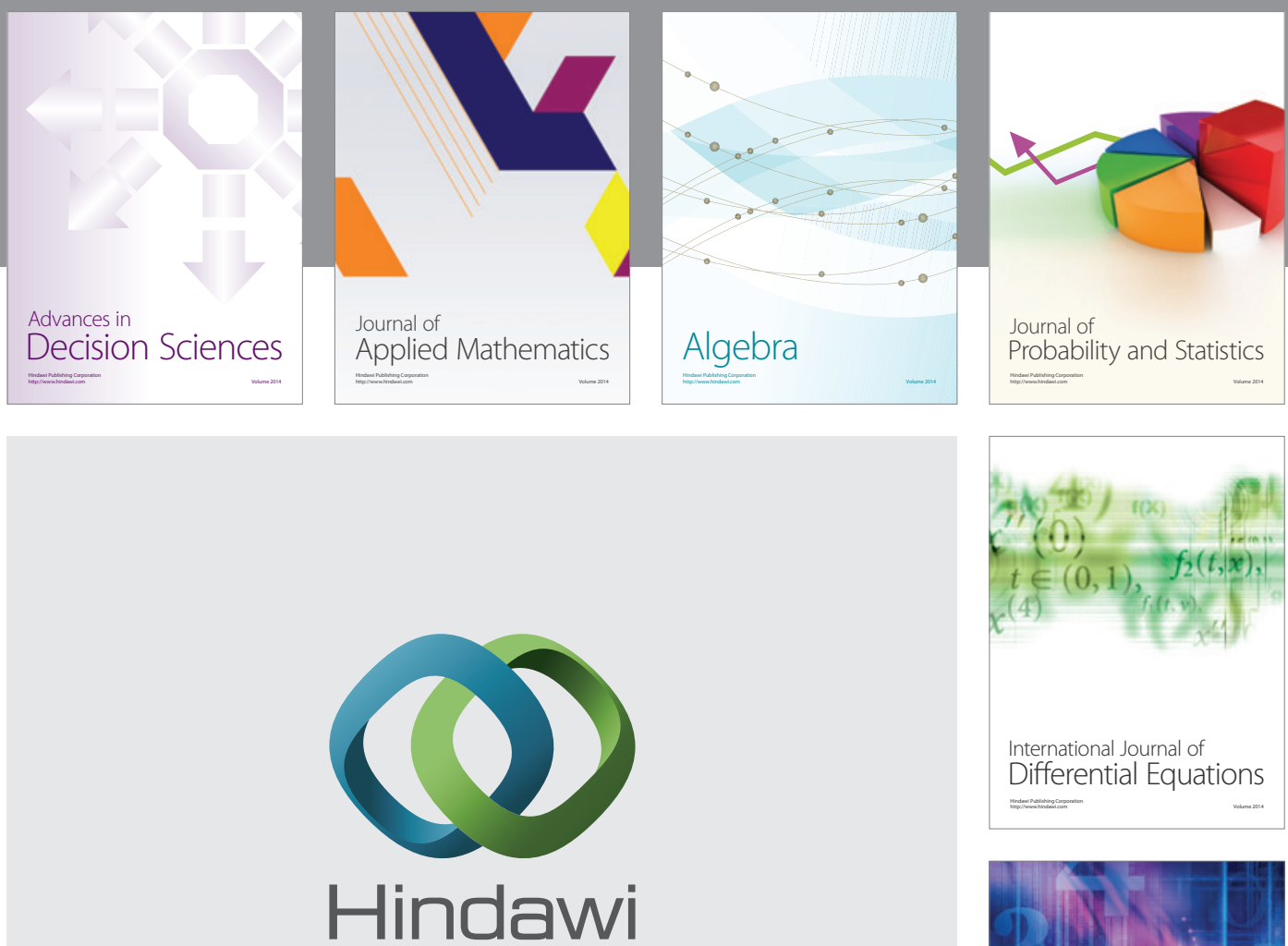

Submit your manuscripts at http://www.hindawi.com
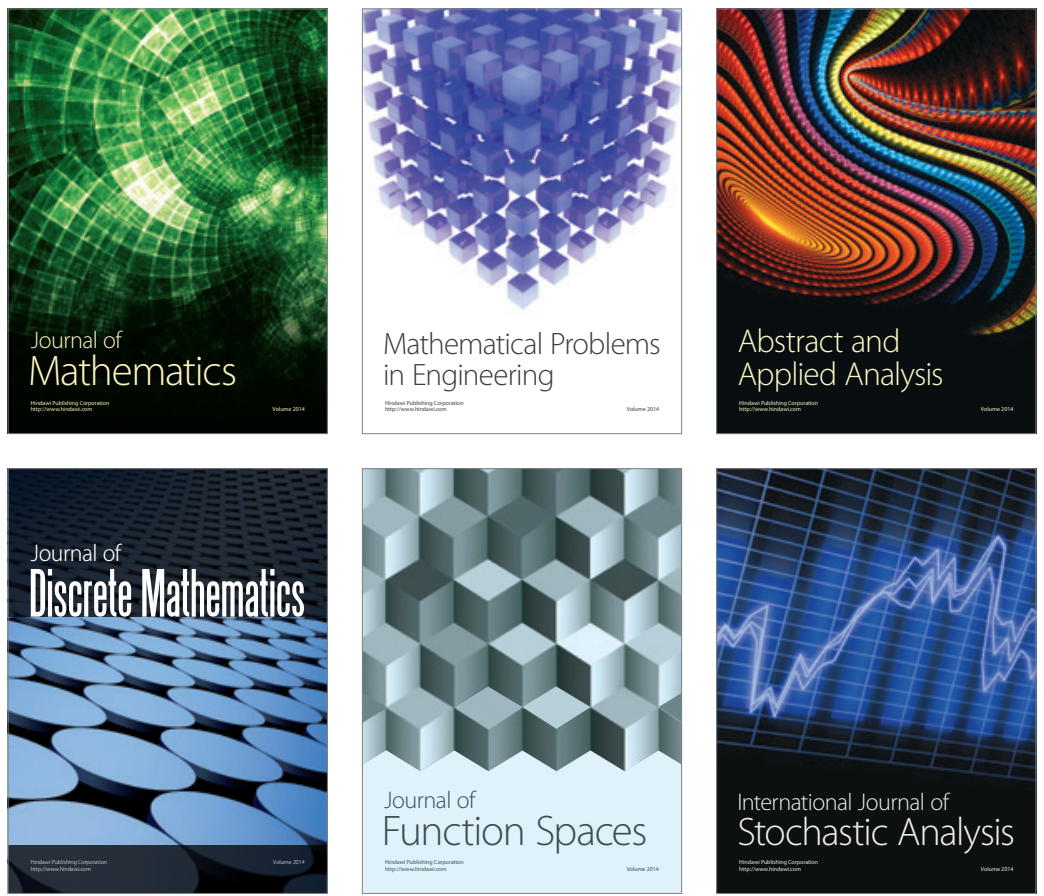

Journal of

Function Spaces

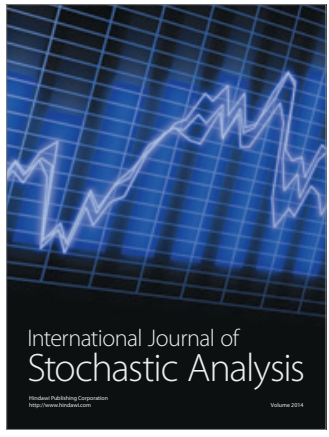

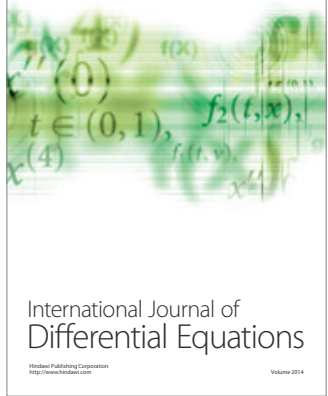
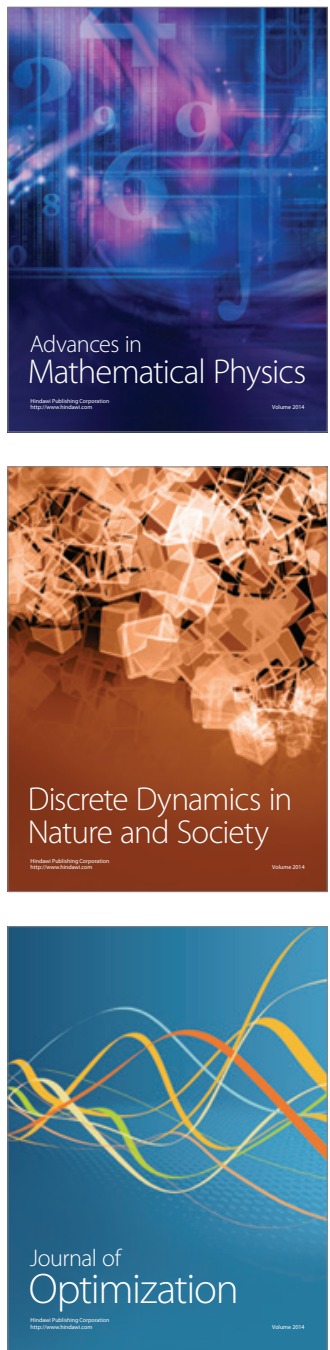